Article

\title{
Functional and Structural Characterization of FAU Gene/Protein from Marine Sponge Suberites domuncula
}

\author{
Dragutin Perina ${ }^{1, \dagger}$, Marina Korolija ${ }^{2, \dagger}$, Marijana Popović Hadžija ${ }^{3}$, Ivana Grbeša ${ }^{4}$, \\ Robert Belužić $^{3}$, Mirna Imešek ${ }^{1}$, Christine Morrow ${ }^{5}$, Melanija Posavec Marjanović ${ }^{1}$, \\ Tatjana Bakran-Petricioli ${ }^{6}$, Andreja Mikoč ${ }^{1, *}$ and Helena Ćetković ${ }^{1, *}$
}

1 Division of Molecular Biology, Ruđer Bošković Institute, Zagreb 10000, Croatia; E-Mails: dperina@irb.hr (D.P.); Mirna.Imesek@irb.hr (M.I.); Melanija.Posavec@irb.hr (M.P.M.)

2 Forensic Science Centre "Ivan Vučetić", Zagreb 10000, Croatia; E-Mail: korolijamm@gmail.com

3 Division of Molecular Medicine, Ruđer Bošković Institute, Zagreb 10000, Croatia; E-Mails: Marijana.Popovic-Hadzija@irb.hr (M.P.H.); Robert.Beluzic@irb.hr (R.B.)

4 The Mina and Everard Goodman Faculty of Life Sciences, Bar-Ilan University, Ramaty-Gan 5290002, Israel; E-Mail: ivana.grbesa3@gmail.com

5 Queen's University Belfast, Marine Laboratory, Portaferry BT22 1PF, Northern Ireland, UK; E-Mail: christinemorrow@gmail.com

6 Department of Biology, Faculty of Science, University of Zagreb, Zagreb 10000, Croatia; E-Mail: tbakran@biol.pmf.hr

$\dagger$ These authors contributed equally to this work.

* Authors to whom correspondence should be addressed; E-Mails: mikoc@irb.hr (A.M.); cetkovic@irb.hr (H.Ć.); Tel.: +385-1-456-1115; Fax: +385-1-456-1177.

Academic Editors: Friedemann Honecker and Sergey A. Dyshlovoy

Received: 6 May 2015 / Accepted: 8 June 2015 / Published: 7 July 2015

\begin{abstract}
Finkel-Biskis-Reilly murine sarcoma virus (FBR-MuSV) ubiquitously expressed (FAU) gene is down-regulated in human prostate, breast and ovarian cancers. Moreover, its dysregulation is associated with poor prognosis in breast cancer. Sponges (Porifera) are animals without tissues which branched off first from the common ancestor of all metazoans. A large majority of genes implicated in human cancers have their homologues in the sponge genome. Our study suggests that FAU gene from the sponge Suberites domuncula reflects characteristics of the $F A U$ gene from the metazoan ancestor, which have changed only slightly during the course of animal evolution. We found pro-apoptotic activity of sponge FAU protein. The same as its human homologue, sponge FAU increases apoptosis
\end{abstract}


in human HEK293T cells. This indicates that the biological functions of FAU, usually associated with "higher" metazoans, particularly in cancer etiology, possess a biochemical background established early in metazoan evolution. The ancestor of all animals possibly possessed FAU protein with the structure and function similar to evolutionarily more recent versions of the protein, even before the appearance of true tissues and the origin of tumors and metastasis. It provides an opportunity to use pre-bilaterian animals as a simpler model for studying complex interactions in human cancerogenesis.

Keywords: ribosomal protein genes; snoRNA; FAU; RPS30; SNORA62; evolution; Porifera

\section{Introduction}

The evolution of cancer is still not fully understood. However, it is known that the genes of cellular cooperation that evolved along with multicellularity, when malfunctioned, contribute to cancer development [1]. The majority of these genes are involved not only in cell division and cell growth, but also in cell adhesion, apoptosis, developmental signaling pathways, recognition of self and non-self and differentiation of various cell types. All living animals descended from the 600 million year old common ancestor, including sponges and humans [2]. Sponges are the simplest animals, but despite the absence of tissues, their genomes contain a large majority of genes that have been implicated in human cancer [2]. However, the functions of these genes and biochemical characteristics of their products within the sponge cells remained largely unknown $[3,4]$. Tumors have not yet been identified in sponges, although computational studies have predicted that most metazoans might be prone to develop tumors [5]. Moreover, naturally occurring tumors have recently been described in two cnidarian species [6]. These findings suggest that understanding of cancer associated genes in simpler animals without tissues and blood vessels could help in understanding the more complex interactions of their homologues in higher Metazoa.

Ribosome assembly is a complex process requiring coordinated activation of more than 200 non-ribosomal factors, including numerous small nucleolar RNAs (snoRNAs). Their role in modification of ribosomal RNAs (rRNAs) was shown to be essential for the correct assembly of the ribosome $[7,8]$. Three major classes of snoRNAs include: C/D box snoRNAs (primarily guide the 2'-O-methylation of target rRNAs), H/ACA box snoRNAs (typically guide pseudouridylation of target rRNAs) and small Cajal-body-specific RNAs (scaRNAs) (typically target snoRNAs). snoRNAs are believed to be the most ancient non-coding RNAs (ncRNAs) [9]. Many examples of ncRNAs displaying both snoRNA and microRNA (miRNA) characteristics suggest a possible evolution from one type to the other [10]. snoRNAs are nonautonomous transposable elements that can gain additional genomic copies, usually by a copy-and-paste mechanism involving an RNA intermediate. These snoRNA retroposons (snoRTs), like Alu element, use the LINE L1 machinery for their mobilization, and probably play an important role in evolution of mammalian genome [11].

Ribosomal proteins (RPs) are an evolutionarily conserved component of ribosome in every cell of every organism. Analysis of 66 complete genomes revealed that 34 RPs are common to all living organisms [12]. Many RPs possess additional extraribosomal functions in cells. They are involved in 
many processes within the ribosome system, surveillance of ribosome synthesis, but also in replication and regulation of cell growth, apoptosis and cancer [13]. Human FAU gene (Finkel-Biskis-Reilly murine sarcoma virus (FBR-MuSV) ubiquitously expressed) encodes the ribosomal protein S30 (RPS30) fused with an ubiquitin-like protein FUBI [14]. Retrovirus FBR-MuSV, which contains genes $v$-fos and fox, can induce osteosarcomas in susceptible mice [15]. Fox is an antisense sequence to the cellular gene $F A U$, which indicates a putative tumor suppressor role for FAU. Moreover, the FAU gene is down-regulated in human breast, prostate and ovarian tumors and its down-regulation is strongly associated with poor prognosis in breast cancer [16-18]. Pro-apoptotic regulatory role for FAU has recently been described [19]. Since the failure of apoptosis is fundamental to the development of many cancers, regulation of apoptosis may serve as the functional background for the extraribosomal function of this RP.

Our study suggests that FAU gene from the sponge Suberites domuncula probably reflects the characteristics of the FAU gene from metazoan ancestor that changed only slightly during animal evolution. FAU protein from sponge possesses pro-apoptotic activity and increases apoptosis in human HEK293T cells, same as its human homologue. This suggests that biological functions of FAU, usually associated with the origin of cancer in "higher" metazoans, possess biochemical background established early in metazoan evolution. Our results implicate that the ancestor of all animals possessed FAU protein with the structure and the function similar to its evolutionarily recent versions, even before the appearance of true tissues and the origin of tumors and metastasis.

\section{Results and Discussion}

\subsection{Structure and Evolution of Metazoan FAU Gene}

FAU gene encodes an ubiquitin-like protein (FUBI) fused to the ribosomal protein S30 (RPS30). This type of gene organization seems to be conserved throughout metazoan evolution (Figure 1A). Sponge FAU protein has $61 \%$ amino acid sequence identity (78\% sequence similarity) with human FAU and is more similar to its homologues from human or rat, than to those from invertebrates Caenorhabditis elegans (47\% sequence identity, 62\% similarity) and Drosophila melanogaster (50\% sequence identity, 68\% similarity). Both invertebrate model organisms underwent recent accelerated evolution [20]. Since sponge FAU changed only slightly during metazoan evolution, it seems to be a promising candidate to effectively reflect FAU protein structure of the ancestral metazoan ribosome. It is also noteworthy that FUBI domain is much less conserved than RPS30. However, G-G dipeptide motif, which participates in bond formation between ubiquitin and lysine residues of target proteins, is conserved in all analyzed metazoans (Figure 1A). As already observed in human FAU, none of the lysine residues which serve as sites for polyubiquitin chain formation, are present in any of the analyzed FAU homologues (Figure 1A). This indicates that FUBI is unlikely to have an analogous role to ubiquitin in protein degradation and that this diverse role was probably established early in metazoan evolution. 
A

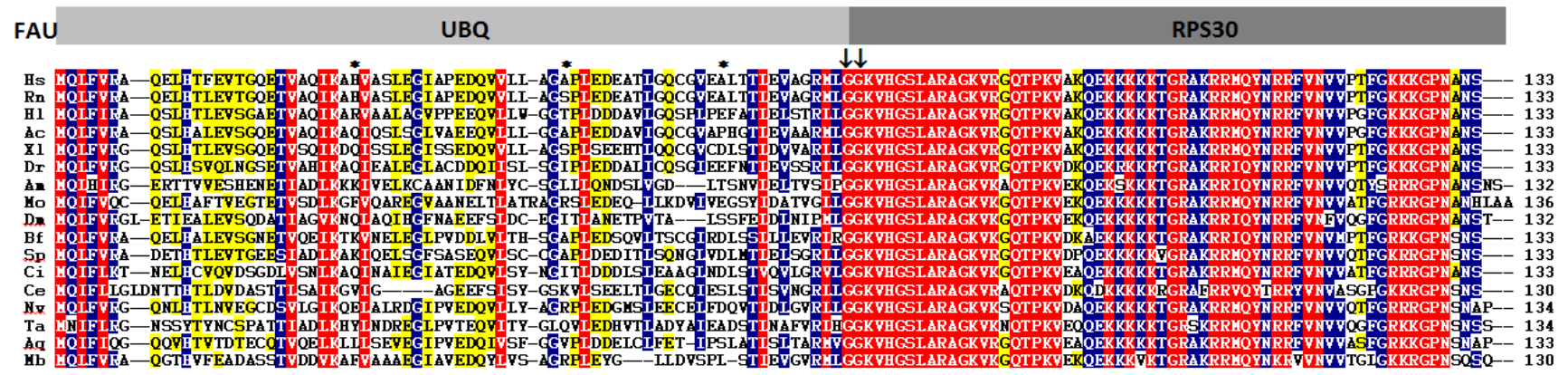

B

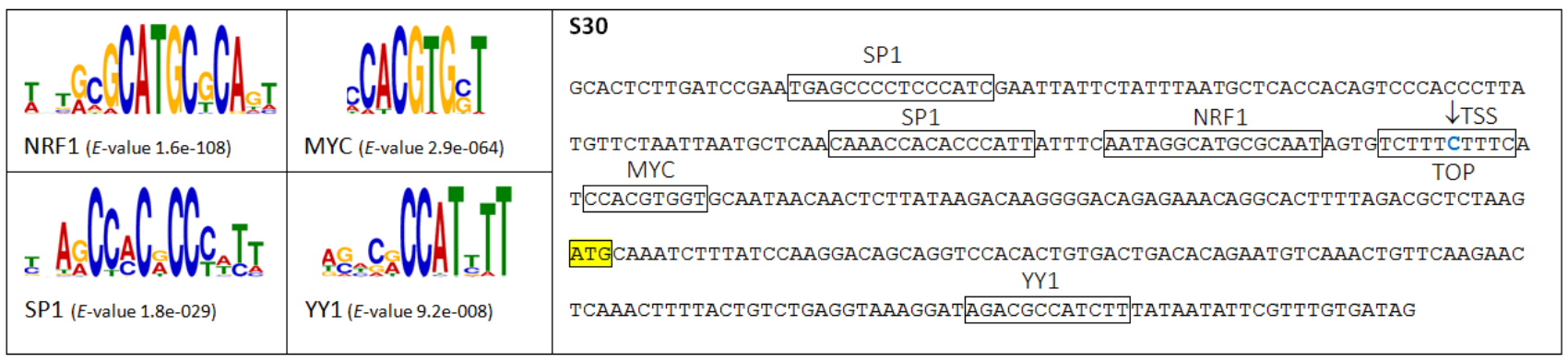

Figure 1. (A) Multiple sequence alignment of FAU proteins from representative species (Homo sapiens (Hs), Rattus norvegicus (Rn), Haliaeetus leucocephalus (H1), Anolis carolinensis (Ac), Xenopus laevis (Xl), Danio rerio (Dr), Apis mellifera (Am), Metaseiulus occidentalis (Mo), Drosophila melanogaster (Dm), Branchiostoma floridae (Bf), Strongylocentrotus purpuratus (Sp), Ciona intestinalis (Ci), Caenorhabditis elegans (Ce), Nematostella vectensis (Nv), Trichoplax adhaerens (Ta), Amphimedon queenslandica (Aq), Monosiga brevicollis (Mb)). G-G dipeptide motif and missing lysine residues which serve as sites for polyubiquitin chain formation are marked with arrows and asterisks, respectively; red $=100 \%$, blue $=80 \%$ and yellow $=60 \%$ identity; $(\mathrm{B})$ Over-represented motifs in sponge ribosomal protein gene (RPG) promoters and promoter sequence of sponge FAU with indicated translational start site (marked with yellow rectangle), transcription start site (TSS, marked with arrow) and transcription factor binding sites and terminal 5'-terminal oligopyrimidine (TOP) tract (marked with rectangles).

Synchronized expression of ribosomal protein genes (RPGs), driven by the promoters of similar strength, ascertains equimolecular amount of ribosomal proteins in the cell, which is prerequisite for the ribosome assembly [21,22]. Human over-represented motifs in RPG promoters are identified as targets for YY1, NRF1, c-Myc and SP1 transcription factors [21]. The 5'-terminal oligopyrimidine (TOP) tract in human RP transcripts is also known to be essential for the control of gene expression, at both the transcriptional and the translational levels [23,24]. To test which elements are present in the FAU promoter, we compared over-represented motifs in RPG promoters of the sponge Amphimedon queenslandica with $F A U$ sequence. In the majority of analyzed human RPG promoters, YY1 motifs were found downstream of the transcription start site (TSS) [25]. This motif is also present downstream in the sponge FAU ( $\left.p=3.00 \times 10^{-7}\right)$ (Figure 1B). Two SP1 and one NRF1 binding sites were found upstream of the TSS $\left(p=4.08 \times 10^{-7}, p=1.39 \times 10^{-7}, p=7.10 \times 10^{-7}\right.$, respectively), 
while E-box palindromic core CACGTG was also identified in FAU promoter $\left(p=3.12 \times 10^{-6}\right)$. Among many transcription factors that recognize the E-box, oncoprotein c-Myc (MYC) is known to bind to it [26]. c-Myc enhances ribosomal biogenesis by up-regulating transcription mediated by all three RNA polymerases [27]. This implies possible participation of the FAU protein in the analogous c-Myc-enhanced ribosomal biogenesis established early in metazoan evolution. The TSS was identified in TOP (Figure 1B). The TOP motif has a dual regulatory function in vertebrates. It is a part of a cis-regulatory element in transcriptional regulation and has a role at the translational level [23,24]. In certain physiological conditions, it inhibits binding of the translational regulatory proteins or the translational machinery to the mRNA [28]. The presence of TOP and all other elements in sponge $F A U$ promoter indicates that the foundations of the human FAU cis-regulation were established early in metazoan evolution.

Multiple sequence alignment of FAU gene orthologues was used to characterize intron dynamics by comparing the intron positions (Figure 2). Most of the positions, phases, and the number of introns in FAU genes were not significantly changed from sponge to human (Figure 2). The most conserved intron position separates the UBQ from RPS30 domain in FAU and varies from 25 to $3339 \mathrm{bp}$. The average value of sponge RPG intron length is $164 \mathrm{bp}$ [29], while human RPGs have significantly longer introns, with the average length of $760 \mathrm{bp}$ [30]. Transposable element insertions play an important role in the evolution of intron size [31]. Therefore, we searched for over-represented elements in FAU introns.

\subsection{Identification of snoRNAs in FAU Introns of Sponges}

The accumulating genomic data strongly confirm the tendency of snoRNAs to colonize RPGs and ribosome related genes in eukaryotes [32]. The total set of 16 introns in FAU gene from eight sponges produced four candidate H/ACA snoRNAs. After a more detailed analysis, we were able to identify three snoRNAs that match a sequence motif of known snoRNAs available on Rfam, the snOPY database and the snoRNA-LBME database. This snoRNA is the sponge orthologue of the human SNORA62 (E2) (Figure 3A). SNORA62 is predicted to guide the pseudouridylation of uridine residues at the position 3830 and 3832 in human 28S rRNA [33]. This target sequence of 28S rRNA is highly conserved between human and analyzed sponges (Figure 3B). The sponge SNORA62 pseudouridylation guide sequence, as well as the $\mathrm{H}$ - and ACA-boxes, are also well conserved (Figure 3A). Its expression was verified experimentally (Figure 3C). Phylogenetic analysis of SNORA62/SNORA6 is presented in Figure 3D. The SNORA62 and SNORA6 share the same host gene in vertebrates and SNORA6 probably arose by cis-duplication of SNORA62. The SNORA62 homologues from sponges clearly group together and reflect the characteristics of snoRNA ancestral to this snoRNA family, before the duplications and diversifications within the metazoan lineage. snoRNAs are mobile genetic elements, often transferred through retrotransposition, and can therefore participate in diversification and enrichment of transcriptomes through various mechanisms such as intron/exon gain/loss [34]. It is known that snoRNAs can change their genomic location within relatively short vertebrate evolutionary time scales [35]. Since all of examined RPG intron positions were conserved in three Suberites species, it was presumed that on this shorter evolutionary time scale, mobility of snoRNAs is not a significant factor that determines intron dynamics [29]. However, we found that within the same genus 
(Suberites), SNORA62 can change its genomic location. In S. pagurorum, S. domuncula and S. ficus this snoRNA is present in the same intron, but apparently is missing in the same conserved intron in S. massa (Figure 2). Our results indicate that RPG introns are more dynamic than previously documented and that mobility of snoRNAs plays an important role in RPG (and FAU) evolution even on shorter evolutionary time scales.

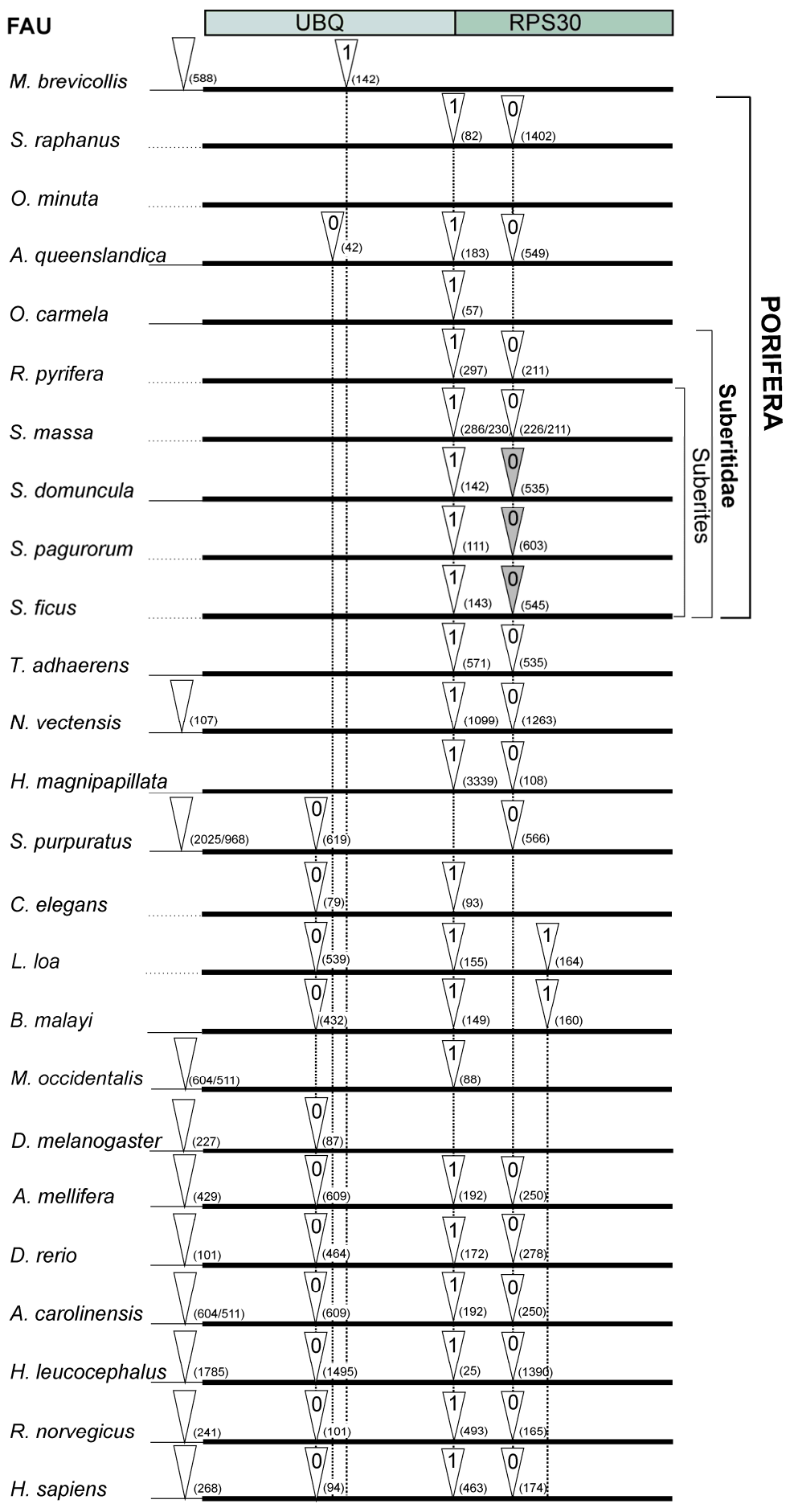

Figure 2. Intron-mapping of $F A U$ genes from representative species. White triangles indicate positions of the introns and gray triangles indicate presence of SNORA62. The number within triangle denotes the intron phase and the number in brackets intron length. The thin line indicates the 5' UTR region. 
A

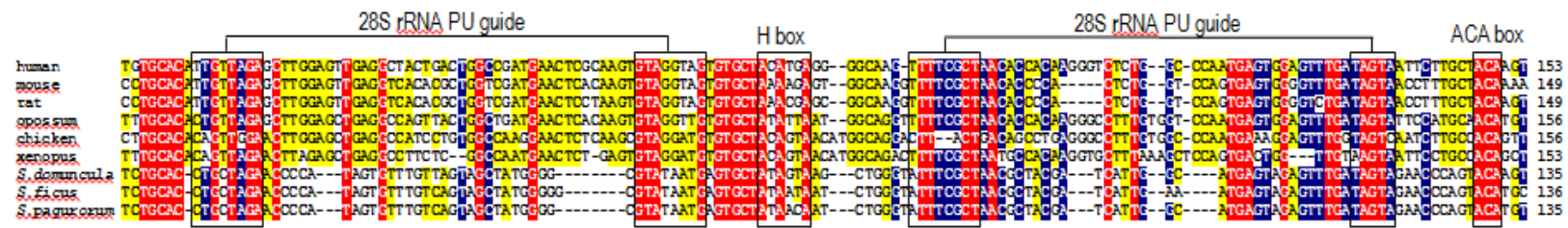

B

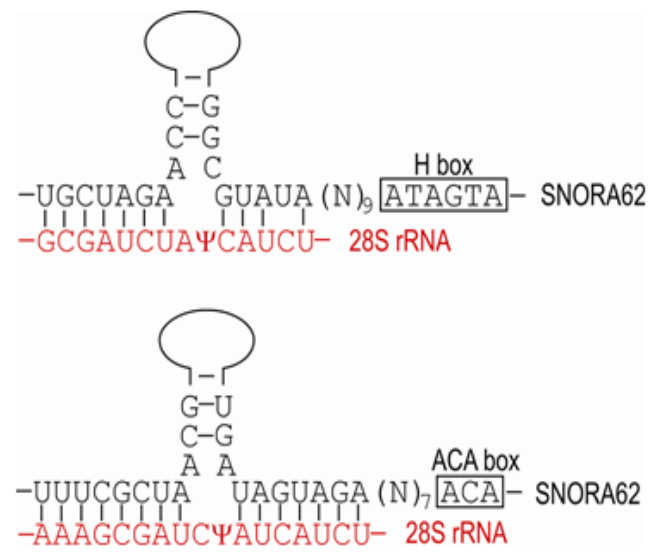

D

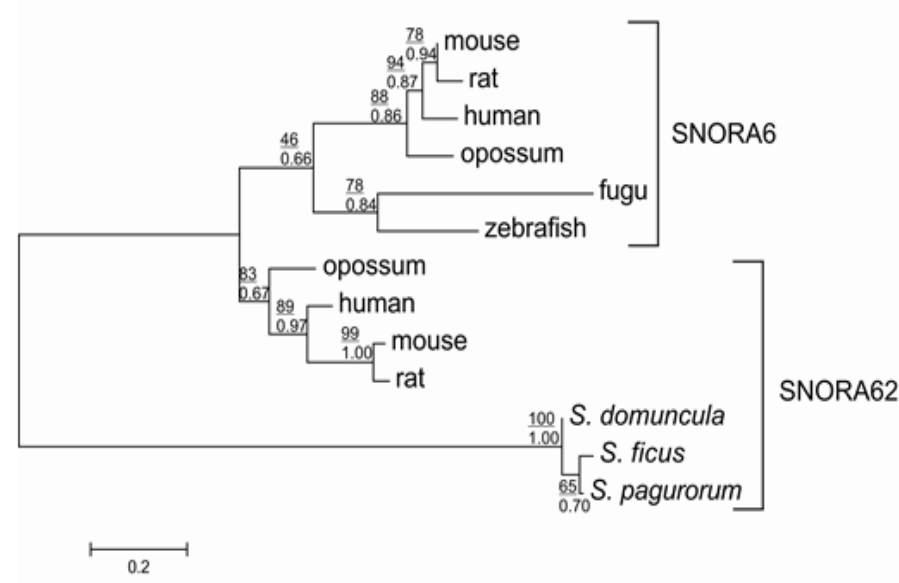

C
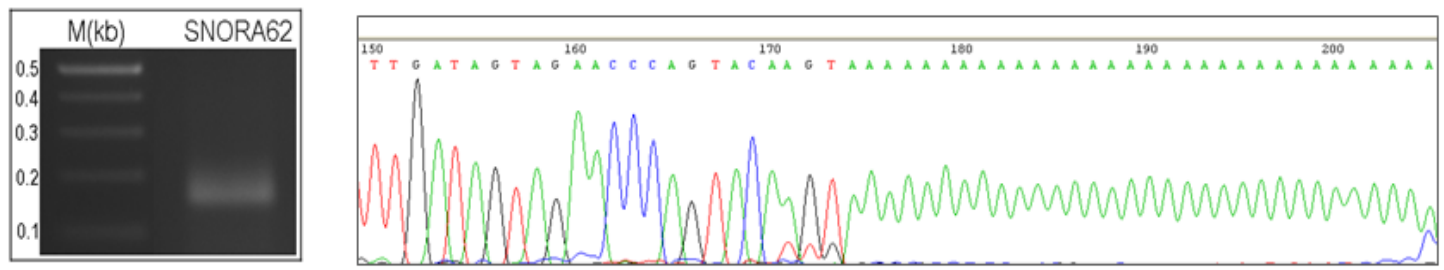

Figure 3. (A) SNORA62 conserved in the last introns of the FAU gene in S. domuncula, S. ficus and S. pagurorum. All essential snoRNA elements are conserved and a putative pseudouridylation $(\mathrm{PU})$ guide site is designated; red $=100 \%$, blue $=80 \%$ and yellow $=60 \%$ identity; (B) Target sites of SNORA62 are conserved in sponges and marked with psi; (C) Experimental verification of transcription of sponge SNORA62. Polyadenylated snoRNAs were amplified, cloned and sequenced; (D) Maximum likelihood (ML) phylogenetic tree of snoRNAs from representative species. Bootstrap values for ML are given above and MCMC below the line. The scale bar indicates the genetic distance of the branch lengths.

The other snoRNA shows stable secondary structure with conserved snoRNA parts (Figure S1). In the first intron of FAU gene of the sponge Rhizaxinella pyrifera, a single snoRNA with a potential target rRNA was found (Figure S1). This target is not conserved in humans nor has it yet been described as a pseudouridylation site, so we can only speculate about the possible function of this snoRNA. 


\subsection{Subcellular Localization of Sponge FAU}

RPs are mainly cytoplasmic, being incorporated into translating ribosomes, but they can be found, at least transiently, in the nucleus [36]. As mentioned before, RPs have non-ribosomal functions when they are off-ribosomal subunits. We transiently transfected HEK293T and HeLa cells with GFP-FAU from S. domuncula and DsRed-FAU from human and analyzed the cells 48 hours post transfection using confocal laser scanning microscopy. Proteins show the same localization pattern in both cell types. Both human and sponge proteins exhibit the same subcellular localization (Figure 4A). Proteins are dispersed throughout the cytoplasm rather than being associated with a specific cytoplasmic organelle (Figure 4B,C). Although the signals are present mainly in the cytoplasm, portions can also be clearly observed in the nuclei (Figure 4A). This result matches the finding of the previous study, where subcellular localization of Drosophila RPS30 was analyzed [36]. It has been observed that a fraction of RPS30 is localized in the nucleus and is associated with transcription sites. We presume that, according to cellular localization, extraribosomal functions of FAU at transcription sites were already present in ancestor of all metazoans and remained conserved throughout the evolution.

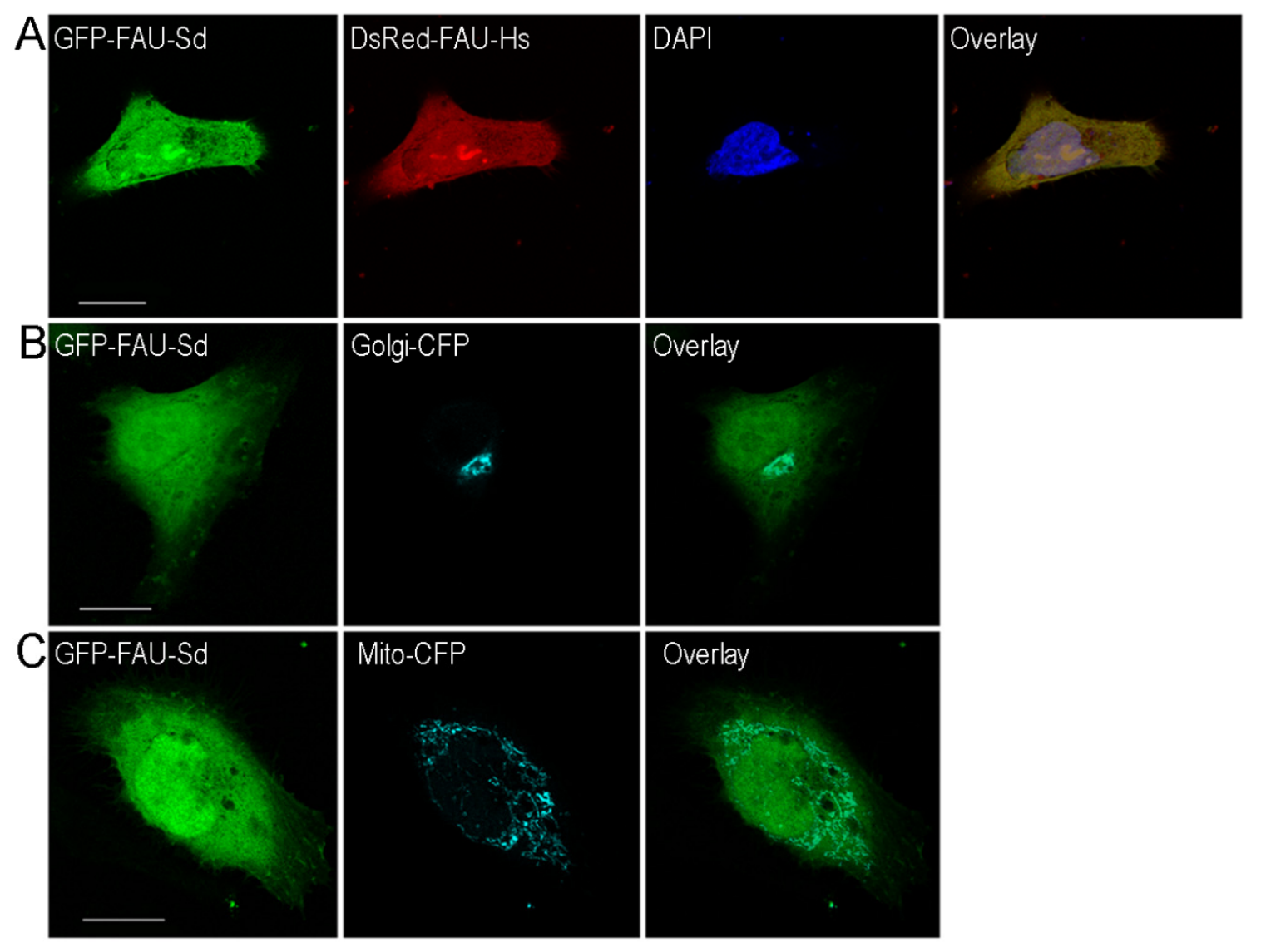

Figure 4. (A) Subcellular localization of sponge and human FAU. HeLa cells transiently transfected with sponge (Sd) pEGFP-FAU (green fluorescence), human (Hs) pDsRed-FAU (red fluorescence); (B) pECFP-Golgi (cyan) and (C) pECFP-mitochondria (cyan). The overlay (yellow) shows colocalization of the human and sponge homologs in panel $\mathbf{A}$. Scale bar $=10 \mu \mathrm{m}$. 

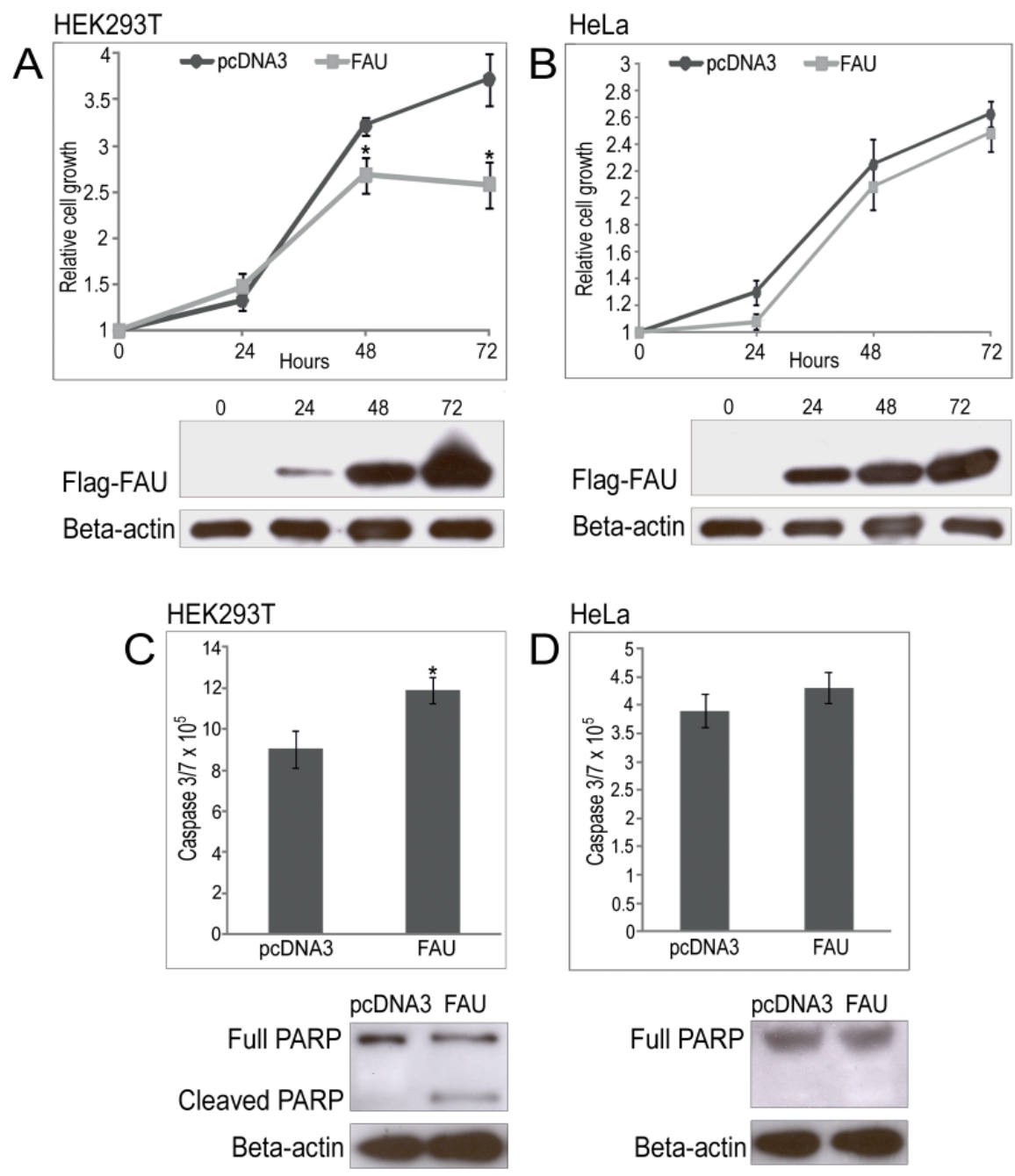

Figure 5. Protein blot validation of sponge Flag-FAU expression and the relative growth rates assessed by CellTiter-Glo assay of HEK293T (A) and HeLa cells (B); Activities of caspase were measured by a Caspase-Glo 3/7 assay kit and by cleavage of caspase substrate PARP in HEK293T (C) and HeLa cells (D) transfected with pcDNA3-FAU or empty vector pcDNA3. Data are representative of three independent experiments $\left({ }^{*} p<0.05\right)$.

\subsection{Overexpression of Sponge FAU in Human Cell Lines}

Transient transfection with human pcDNA3-FAU increased apoptosis and decreased short-term cell viability in HEK293T, Jurkat and CEM cell lines [19]. To test whether these functions of FAU protein were established early in metazoan evolution, we transfected HEK293T and HeLa cell lines with sponge pcDNA3-FAU. The growth rate of cells was evaluated at different time points after transfection, to determine whether overexpression of sponge FAU affects cell proliferation. Transfection markedly increased Flag-FAU levels (Figure 5A,B). Empty vector pcDNA3 was used as control. Subsequently, the proliferation of pcDNA3-FAU-transfected HEK293T cells was decreased 1.44-fold ( $p=0.008)$ when compared to cells with pcDNA3 control (Figure 5A). Cell viability decreased by $30.1 \% 72 \mathrm{~h}$ post-transfection compared to cells possessing the control vector. Interestingly, these effects were not observed in HeLa cancer cell line (Figure 5A). To further investigate the mechanism underlying the observed, we measured caspase- 3 and -7 activities in transfected cell lines. pcDNA3-FAU-transfected 
HEK293T cells show 1.31-fold $(p=0.014)$ increased caspase activity as compared to pcDNA3 transfected cells (Figure 5C). However, apoptotic cells were not elevated in HeLa cell line, and neither the cleavage of PARP, caspase-3 substrate, was observed (Figure 5D). Interestingly, similar results were obtained when human Flag-FAU was overexpressed in HeLa cells (Figure S2). Our results indicate that pro-apoptotic activity of FAU was established early in metazoan evolution, but appears to be cell type specific as its expression leads to efficient apoptosis in HEK293T but not in HeLa cell line. Since both human and sponge FAU do not activate caspases in HeLa cells significantly, but both activate caspases in HEK293T cells, we believe that the mechanism underlying this effect was already present in metazoan ancestor. It was proposed that Bcl-G plays an essential down-stream role in mediating the pro-apoptotic activity of FAU [19]. The molecular mechanisms of apoptosis regulation by FAU remain to be elucidated, but possibly include FUBI-mediated targeting of Bcl-G and/or modulation of the interaction of Bcl-G with other constituents of the cellular apoptotic machinery (caspases for example) [19]. Although down-regulation of Bcl-G inhibits apoptosis induced by FAU, basal apoptosis rates are higher in certain cellular contexts [19]. However, this effect has not been observed in human breast cancer and prostate cell lines [16,17]. This indicates that in certain cell lines, Bcl-G can have anti-apoptotic effects, in addition to the pro-apoptotic activity. Moreover, mouse Bcl-G is expressed in a range of epithelial, as well as in dendritic cells and its loss does not appear to affect any of these cell types [37]. This intriguing contrast in the effects of FAU/Bcl-G on cell fate in different cellular contexts presents attractive possibilities for the development of novel therapies for cancers and could be used for targeted elimination of cancer cells.

From all presented data we can conclude that the structure and the functions of the recent $F A U$ gene were established early in metazoan lineage. Tumor suppressor gene function of FAU probably arose before tumor appearance. As sponges do not possess tissues and organs, formation of tumors within them is unlikely. It seems that tumors evolved in parallel with the development of true tissues and organs, yet the extraribosomal functions of RPs were probably established in the metazoan ancestor well before the Cambrian explosion, i.e., before the appearance of diverse groups of multicellular animals. Other highly sophisticated extraribosomal functions of RPs, involved in the origin of cancer and metastasis, evolved together with the appearance of different cell types, tissues and organs. Whereas synchronized cis-regulation of RPGs needs to be conserved for equimolecular presence of RPs in the metazoan cell, the evolution of RPGs together with the extraribosomal functions of RPs on shorter evolutionary time scale is probably driven by snoRNAs. snoRNAs can promote, but also suppress tumor development [38]. Numerous studies already provided evidence for the functional importance of snoRNAs in cancerogenesis [39,40]. Our results show that SNORA62 is found in FAU gene in the subset of sponges from the genus Suberites, which suggests its dynamic and fast evolving nature. This snoRNA is located in the human ribosomal protein gene SA (RPSA). Both host RPGs possess an extraribosomal function involved in the maintenance of cellular viability through the caspase-dependent regulation of apoptosis [19,41]. Interestingly, SNORA62 is the most upregulated gene in diallyl sulfide (DAS)-induced apoptosis in HeLa cells, as a part of p53, mitochondria- and caspase- pathways [42]. It would be interesting to test whether this snoRNA can act as an oncogene like SNORA42 [43], or tumor suppressor like U50 snoRNA [44] and if these functions interfere and modulate similar functions of its host genes. The number of ncRNA is exceedingly lower in basal metazoans than in "higher" animals, which may indicate that metazoan complexity correlates with an 
increasing number of ncRNAs [45]. If SNORA62 can modulate apoptotic effect of its host genes, it will provide new strategies for targeting FAU. In the longer term, the development of techniques for the manipulation of snoRNAs may also be productively directed towards the manipulation of genes involved in cancerogenesis for cancer therapy. Analyses of sponge FAU gene presented in our paper provides the opportunity to determine direction for further investigation of FAU and evaluate its potential for cancer treatment. Undoubtedly, sponges could be used as an informative model in cancer research, not only in terms of targeting genes involved in cancerogenesis by snoRNAs, but also in targeting snoRNAs themselves. In order to evaluate their potential for cancer treatment, further investigation of highly conserved and mobile snoRNAs found in the metazoan common ancestor is needed.

\section{Experimental Section}

\subsection{Materials}

Live specimens of the sponge Suberites domuncula (Porifera, Demospongiae, Tetractinomorpha, Hadromerida, Suberitidae) and Sycon raphanus (Porifera, Calcarea, Calcaronea, Leucosolenida, Sycettidae) were collected in the Northern Adriatic Sea near Rovinj, Croatia, and stored at $-80{ }^{\circ} \mathrm{C}$. Specimens of Oopsacas minuta (Porifera, Hexactinellida, Hexasterophora, Lyssacinosida, Leucopsacidae) were collected in the cold water pit in the Middle Adriatic and specimens of Rhizaxinella pyrifera (Porifera, Demospongiae, Tetractinomorpha, Hadromerida, Suberitidae) were collected in the Middle Adriatic Pit, around $200 \mathrm{~m}$ depth and stored in 96\% EtOH. Suberites ficus and Suberites pagurorum were collected in Ireland, while Suberites massa in the English Channel.

\subsection{Sequence Analyses}

Homology searches and sequence retrievals were done using BLAST (NCBI, NIH, Bethesda, MD, USA: http://www.ncbi.nlm.nih.gov). Sequences were analyzed using Lasergene (DNAStar, Madison, WI, USA). Multiple sequence alignments (MSA) were performed with CLUSTALX [46]. The exact position and the phase of each intron were verified by manual inspection and statistical data were extracted from GeneDoc (http://www.psc.edu/biomed/genedoc).

Over-represented motifs were searched using MEME [47], as described earlier [22]. Statistical significance of the motif ( $E$-value) and $p$-value (statistical significance of predicted sites in $F A U$ ) were extracted from MEME. The location of transcription factor binding sites relative to the transcription start site was also extracted from MEME results for FAU sequence within a set of sequences with the same identified motif. Obtained motifs were then compared using TOMTOM [48] against the TRANSFAC database of known motifs [49].

\subsection{Isolation of Genomic DNA and Sequencing of FAU Gene}

For genomic DNA preparation, sponge specimens were cut into pieces, frozen in liquid nitrogen and grounded to fine powder from which total DNA was isolated using the Genomic DNA Purification kit (QIAGEN, Redwood City, CA, USA). Sponge FAU gene was amplified by PCR, using LA Taq DNA polymerase (Takara, Japan) and primers specific for ends of the coding sequence of: 
Demospongiae (5'-GCAAGTTTTCGTACAAGGAGGGGC-3' and 5'-GGTGCATTTGAGTTTGGTC CAC-3'), Hexactinellida (5'-ATGCAAGTGTTTGCACAAAC-3' and 5'-CTAACTAGCTGAGTTTGA G-3') and Calcarea (5'-CAGATTTTCATTCAGGCCCAGGC-3' and 5'-GCGTTAGAGTTGGGTC-3'). The amplified fragments were separated on a $0.8 \%$ agarose gel, purified and cloned into the pGEM-T vector (Promega, Madison, WI, USA). Positive clones were sequenced using T7/pUC primers.

\subsection{Isolation and Characterization of Small RNAs}

snoSeeker was used to identify snoRNAs in introns of FAU genes [50]. Rfam [51], snOPY (http://snoopy.med.miyazaki-u.ac.jp/) and the snoRNA-LBME databases [52] were used to check whether snoRNAs match to any of the known snoRNAs' motifs. The secondary structure of snoRNA was computed using the RNAfold program of the Vienna RNA Package [53]. Isolation of small RNAs from sponge was described previously [29]. In brief, fresh specimens of $S$. domuncula were cut into pieces, frozen in liquid nitrogen and grounded to a fine powder. mirPremier microRNA Isolation Kit (Sigma, St. Louis, MO, USA) was used, according to the manufacturer's protocol for plant tissue. Polyadenylation was performed by incubation with E. coli Poly(A) Polymerase (BioLabs). Poly(A) tailing reaction mixture was then reverse transcribed using the SuperScript II Reverse Transcriptase (Invitrogen, Waltham, MA, USA) and a modified poly-d(T) primer (5'-GCGTAAGTGA CTAGCGTGTTTTTTTTTTTTVN-3'). The resulting cDNA was used for PCR with a reverse primer (5'-GCGTAAGTGACTAGCGTG-3') and forward primer specific for predicted SNORA62 (5'-CCCCATAGTGTTTGTTAGTAGC-3'). The product was cloned into the pGEM-T vector (Promega). Positive clones were sequenced using the ABI PRISM BigDye Terminator v3.1 Ready Reaction Cycle Sequencing Kit and T7/pUC primers.

For phylogenetic analyses, multiple alignments were performed with CLUSTALW Ver. 1.6 [54]. Aligned sequences were imported into MEGA version 6 [55], and analyzed by Maximum Likelihood (ML), while the Bayesian MCMC analysis was conducted in MrBayes v. 3.1.2. [56]. Bootstrap tests were performed with 1000 replicates. The model for ML analysis was selected using Modeltest 3.7 and the Akaike Information Criterion (AIC) [57], which indicated Hasegawa-Kishino-Yano model (I + G) [58].

\subsection{Cell Culture}

Human HeLa cell line (ATCC) and HEK293T cell line were cultured in L-glutamine DMEM (Invitrogene) supplemented with 10\% fetal bovine serum (FBS, Invitrogene) and GA-1000 (30 mg/mL Gentamicin and $15 \mu \mathrm{g} / \mathrm{mL}$ Amphotericin) (Lonza, Basel, Switzerland) at a 1:1000 ratio in humidified chamber with $5 \% \mathrm{CO}_{2}$ at $37{ }^{\circ} \mathrm{C}$.

\subsection{Plasmid Constructions}

Eukaryotic expression vector pcDNA3 (Invitrogen) was digested with BamHI and EcoRI. The insert containing the FLAG sequence and sponge FAU was cloned using these sets of primers: 5'GTCTAGGGATCCACGAGATGGACTACAAGGACGACGACGATAAGATGCAAGTTTTCGTA-3' and 5'-CTAGACGAATTCTCATCACTGAGGTGCATTTG-3', and human FAU using: 5'GTCTAGGGATCCACGAGATGGACTACAAGGACGACGACGATAAGATGCAGCTCTTTGTC-3' 
and 5'-CTAGACGAATTCTTATTAAGAGTTGGCATTGG-3' For localization assay, sponge FAU was cloned in fusion with GFP using pEGFP-C1 (XhoI/BamHI restriction sites) with the following set of primers: 5'-CCACTCGAGCTATGCAAGTTTTCGTACAAGG-3' and 5'-GACGGATCCTCAT CACTGAGGTGCATTTG-3', and human using pDsRed-C1 with 5'-CCACTCGAGCTATGCAGCT CTTTGTCCGCGC-3' and 5'-ACAGGATCCTTAAGAGTTGGCATTGGGGCCC-3' set of primers.

\subsection{Transient Transfections and Laser Scanning Confocal Microscopy}

Lipofectamine 3000 reagent (Invitrogen) was used for HeLa cells transfections, according to the manufacturer's instructions. Twenty four hours before transfection $5 \times 10^{4}$ cells were seeded onto 24-well culture slides containing DMEM supplemented with 10\% FBS to obtain $80 \%$ confluence. Cells were transfected with $500 \mathrm{ng}$ of plasmid DNA. Forty eight hours post transfection, the cells were washed with PBS pH 7.5, fixed in 4\% formaldehyde, and mounted in SlowFade Diamond Antifade Mountant with or without DAPI (Molecular Probes, Waltham, MA, USA).

Fluorescent images were obtained by Leica SP8 X FLIM laser scanning confocal microscopy equipped with HC PL APO CS2 63×/1.40 OIL objective. GFP was excited by $488 \mathrm{~nm}$ laser line, CFP using $433 \mathrm{~nm}$, DAPI using $405 \mathrm{~nm}$ and DsRed at $560 \mathrm{~nm}$ laser lines.

\subsection{Immunoblotting}

The cell lysates were loaded on SDS-PAGE and electrotransferred to a PVDF Hybond-P membrane (Amersham Biosciences, Piscataway, NJ, USA). The membranes were incubated with anti-FLAG M2 antibody (1:5000) (Sigma) for detection of FLAG-FAU or anti-PARP antibody (1:5000) (ab137653). Protein bands were visualized using chemiluminescence detection (Amersham ECL Plus, GE Healthcare, Parramatta, Australia).

\subsection{Cell Viability Assay}

HeLa cells were transfected as described above, trypsinized $24 \mathrm{~h}$ post-transfection and $10^{3}$ viable cells suspended in $100 \mu \mathrm{L} 10 \%$ FBS DMEM were added to each well of 96-well plates. HEK293T cells were transfected using standard calcium phosphate method [59]. Cell proliferation was measured using CellTiter-Glo Luminescent Cell Viability Assay (Promega) according to the manufacturer's instructions with luminometer Infinite 200 (TECAN). Concentration of ATP was measured in triplicate.

\subsection{Apoptosis Assay}

Cells were transfected as described above, trypsinized $24 \mathrm{~h}$ post-transfection and $5 \times 10^{3}$ viable cells suspended in $100 \mu \mathrm{L} 10 \%$ FBS DMEM were seeded in 96-well culture plates. Caspase-3/7 activity was measured using the Caspase-Glo 3/7 Assay Kit (Promega) according to the manufacturer's instructions with luminometer Infinite 200 (TECAN).

\subsection{Statistical Analyses}

Statistical analysis was performed using Student's $t$-test. Probabilities of less than 0.05 were considered statistically significant. 


\section{Acknowledgment}

This work was supported by the Unity through Knowledge Fund (grant number UKF 1B 2/13) (Andreja Mikoč), Croatian Ministry of Science, Education and Sports grant 098-0982913-2478 (Helena Ćetković) and FP7-REGPOT-2012-2013-1, Grant Agreement Number 316289-InnoMol.

Tatjana Bakran-Petricioli is grateful to Marco Taviani, National Research Council Research Director at ISMAR Institute of Marine Sciences, Bologna, Italy for providing her opportunity to participate in the ARCO (AdRiatic COrals) cruise (13-22 December 2008; in the scope of HERMES Hotspot Ecosystem Research on the Margins of the European Seas project) aboard Research Vessel Urania, for which he was the scientific leader.

\section{Author Contributions}

Live specimens of the sponges were obtained by Christine Morrow and Tatjana Bakran-Petricioli. Andreja Mikoč, Helena Ćetković and Dragutin Perina carried out the molecular genetic studies and the sequence analyses. Andreja Mikoč, Marina Korolija, Christine Morrow, Mirna Imešek, Melanija Posavec Marjanović and Dragutin Perina participated in genomic DNA preparation, sequencing and cloning of FAU. Marina Korolija, Marijana Popović Hadžija, Ivana Grbeša, Robert Belužić and Dragutin Perina carried out colocalization, proliferation, caspase assays. Andreja Mikoč, Helena Ćetković and Dragutin Perina conceived and oversaw the project. Marina Korolija, Andreja Mikoč, Helena Ćetković and Dragutin Perina wrote the manuscript. All authors read and approved the final manuscript.

\section{Conflicts of Interest}

The authors declare no conflict of interest.

\section{References}

1. Davies, P.C.; Lineweaver, C.H. Cancer tumors as Metazoa 1.0: Tapping genes of ancient ancestors. Phys. Biol. 2011, 8, 015001.

2. Srivastava, M.; Simakov, O.; Chapman, J.; Fahey, B.; Gauthier, M.E.; Mitros, T.; Richards, G.S.; Conaco, C.; Dacre, M.; Hellsten, U.; et al. The Amphimedon queenslandica genome and the evolution of animal complexity. Nature 2010, 466, 720-726.

3. Perina, D.; Bosnar, M.H.; Bago, R.; Mikoc, A.; Harcet, M.; Dezeljin, M.; Cetkovic, H. Sponge non-metastatic Group I NME gene/protein-structure and function is conserved from sponges to humans. BMC Evol. Biol. 2011, 11, 87.

4. Perina, D.; Bosnar, M.H.; Mikoc, A.; Muller, W.E.; Cetkovic, H. Characterization of NME6-like gene/protein from marine sponge Suberites domuncula. Naunyn Schmiedeberg Arch. Pharmacol. 2011, 384, 451-460.

5. Domazet-Loso, T.; Tautz, D. Phylostratigraphic tracking of cancer genes suggests a link to the emergence of multicellularity in metazoa. BMC Biol. 2010, 8, 66.

6. Domazet-Loso, T.; Klimovich, A.; Anokhin, B.; Anton-Erxleben, F.; Hamm, M.J.; Lange, C.; Bosch, T.C. Naturally occurring tumours in the basal metazoan Hydra. Nat. Commun. 2014, 5, 4222 . 
7. Perry, R.P. Balanced production of ribosomal proteins. Gene 2007, 401, 1-3.

8. Kressler, D.; Hurt, E.; Bassler, J. Driving ribosome assembly. Biochim. Biophys. Acta 2010, 1803, 673-683.

9. Gardner, P.P.; Bateman, A.; Poole, A.M. SnoPatrol: How many snoRNA genes are there? J. Biol. 2010, $9,4$.

10. Scott, M.S.; Ono, M. From snoRNA to miRNA: Dual function regulatory non-coding RNAs. Biochimie 2011, 93, 1987-1992.

11. Weber, M.J. Mammalian small nucleolar RNAs are mobile genetic elements. PLoS Genet. 2006, 2, e205.

12. Lecompte, O.; Ripp, R.; Thierry, J.C.; Moras, D.; Poch, O. Comparative analysis of ribosomal proteins in complete genomes: An example of reductive evolution at the domain scale. Nucleic Acids Res. 2002, 30, 5382-5390.

13. Warner, J.R.; McIntosh, K.B. How common are extraribosomal functions of ribosomal proteins? Mol. Cell 2009, 34, 3-11.

14. Kas, K.; Michiels, L.; Merregaert, J. Genomic structure and expression of the human FAU gene: Encoding the ribosomal protein S30 fused to a ubiquitin-like protein. Biochem. Biophys. Res. Commun. 1992, 187, 927-933.

15. Michiels, L.; van der Rauwelaert, E.; van Hasselt, F.; Kas, K.; Merregaert, J. FAU cDNA encodes a ubiquitin-like-S30 fusion protein and is expressed as an antisense sequence in the Finkel-Biskis-Reilly murine sarcoma virus. Oncogene 1993, 8, 2537-2546.

16. Pickard, M.R.; Green, A.R.; Ellis, I.O.; Caldas, C.; Hedge, V.L.; Mourtada-Maarabouni, M.; Williams, G.T. Dysregulated expression of FAU and MELK is associated with poor prognosis in breast cancer. Breast Cancer Res. 2009, 11, R60.

17. Pickard, M.R.; Edwards, S.E.; Cooper, C.S.; Williams, G.T. Apoptosis regulators FAU and Bcl-G are down-regulated in prostate cancer. Prostate 2010, 70, 1513-1523.

18. Moss, E.L.; Mourtada-Maarabouni, M.; Pickard, M.R.; Redman, C.W.; Williams, G.T. FAU regulates carboplatin resistance in ovarian cancer. Genes Chromosomes Cancer 2010, 49, 70-77.

19. Pickard, M.R.; Mourtada-Maarabouni, M.; Williams, G.T. Candidate tumour suppressor FAU regulates apoptosis in human cells: An essential role for Bcl-G. Biochim. Biophys. Acta 2011, 1812, 1146-1153.

20. Alba, M.M.; Castresana, J. Inverse relationship between evolutionary rate and age of mammalian genes. Mol. Biol. Evol. 2005, 22, 598-606.

21. Roepcke, S.; Zhi, D.; Vingron, M.; Arndt, P.F. Identification of highly specific localized sequence motifs in human ribosomal protein gene promoters. Gene 2006, 365, 48-56.

22. Perina, D.; Korolija, M.; Roller, M.; Harcet, M.; Jelicic, B.; Mikoc, A.; Cetkovic, H. Over-represented localized sequence motifs in ribosomal protein gene promoters of basal metazoans. Genomics 2011, 98, 56-63.

23. Meyuhas, O. Synthesis of the translational apparatus is regulated at the translational level. Eur. J. Biochem. 2000, 267, 6321-6330. 
24. Shibui-Nihei, A.; Ohmori, Y.; Yoshida, K.; Imai, J.; Oosuga, I.; Iidaka, M.; Suzuki, Y.; Mizushima-Sugano, J.; Yoshitomo-Nakagawa, K.; Sugano, S. The 5' terminal oligopyrimidine tract of human elongation factor $1 A-1$ gene functions as a transcriptional initiator and produces a variable number of US at the transcriptional level. Gene 2003, 311, 137-145.

25. Perry, R.P. The architecture of mammalian ribosomal protein promoters. BMC Evol. Biol. 2005, 5,15 .

26. Van Riggelen, J.; Yetil, A.; Felsher, D.W. MYC as a regulator of ribosome biogenesis and protein synthesis. Nat. Rev. Cancer 2010, 10, 301-309.

27. Oskarsson, T.; Trumpp, A. The Myc trilogy: Lord of RNA polymerases. Nat. Cell Biol. 2005, 7, 215-217.

28. Yamashita, R.; Suzuki, Y.; Takeuchi, N.; Wakaguri, H.; Ueda, T.; Sugano, S.; Nakai, K. Comprehensive detection of human terminal oligo-pyrimidine (TOP) genes and analysis of their characteristics. Nucleic Acids Res. 2008, 36, 3707-3715.

29. Perina, D.; Korolija, M.; Mikoc, A.; Roller, M.; Plese, B.; Imesek, M.; Morrow, C.; Batel, R.; Cetkovic, H. Structural and functional characterization of ribosomal protein gene introns in sponges. PLoS ONE 2012, 7, e42523.

30. Yoshihama, M.; Uechi, T.; Asakawa, S.; Kawasaki, K.; Kato, S.; Higa, S.; Maeda, N.; Minoshima, S.; Tanaka, T.; Shimizu, N.; et al. The human ribosomal protein genes: Sequencing and comparative analysis of 73 genes. Genome Res. 2002, 12, 379-390.

31. Sela, N.; Kim, E.; Ast, G. The role of transposable elements in the evolution of non-mammalian vertebrates and invertebrates. Genome Biol. 2010, 11, R59.

32. Dieci, G.; Preti, M.; Montanini, B. Eukaryotic snoRNAs: A paradigm for gene expression flexibility. Genomics 2009, 94, 83-88.

33. Ganot, P.; Caizergues-Ferrer, M.; Kiss, T. The family of box ACA small nucleolar RNAs is defined by an evolutionarily conserved secondary structure and ubiquitous sequence elements essential for RNA accumulation. Genes Dev. 1997, 11, 941-956.

34. Luo, Y.; Li, S. Genome-wide analyses of retrogenes derived from the human box H/ACA snoRNAs. Nucleic Acids Res. 2007, 35, 559-571.

35. Bompfunewerer, A.F.; Flamm, C.; Fried, C.; Fritzsch, G.; Hofacker, I.L.; Lehmann, J.; Missal, K.; Mosig, A.; Muller, B.; Prohaska, S.J.; et al. Evolutionary patterns of non-coding RNAs. Theory Biosci. 2005, 123, 301-369.

36. Brogna, S.; Sato, T.A.; Rosbash, M. Ribosome components are associated with sites of transcription. Mol. Cell 2002, 10, 93-104.

37. Giam, M.; Okamoto, T.; Mintern, J.D.; Strasser, A.; Bouillet, P. Bcl-2 family member Bcl-G is not a proapoptotic protein. Cell Death Dis. 2012, 3, e404.

38. Williams, G.T.; Farzaneh, F. Are snoRNAs and snoRNA host genes new players in cancer? Nat. Rev. Cancer 2012, 12, 84-88.

39. Mannoor, K.; Liao, J.; Jiang, F. Small nucleolar RNAs in cancer. Biochim. Biophys. Acta 2012, $1826,121-128$.

40. Esteller, M. Non-coding RNAs in human disease. Nat. Rev. Genet. 2011, 12, 861-874.

41. Moodley, K.; Weiss, S.F. Down-regulation of the non-integrin laminin receptor reduces cellular viability by inducing apoptosis in lung and cervical cancer cells. PLoS ONE 2013, 8, e57409. 
42. Wu, P.P.; Chung, H.W.; Liu, K.C.; Wu, R.S.; Yang, J.S.; Tang, N.Y.; Lo, C.; Hsia, T.C.; Yu, C.C.; Chueh, F.S.; et al. Diallyl sulfide induces cell cycle arrest and apoptosis in HeLa human cervical cancer cells through the p53, caspase- and mitochondria-dependent pathways. Int. J. Oncol. 2011, 38, 1605-1613.

43. Mei, Y.P.; Liao, J.P.; Shen, J.; Yu, L.; Liu, B.L.; Liu, L.; Li, R.Y.; Ji, L.; Dorsey, S.G.; Jiang, Z.R.; et al. Small nucleolar RNA 42 acts as an oncogene in lung tumorigenesis. Oncogene 2012, 31, 2794-2804.

44. Dong, X.Y.; Rodriguez, C.; Guo, P.; Sun, X.; Talbot, J.T.; Zhou, W.; Petros, J.; Li, Q.; Vessella, R.L.; Kibel, A.S.; et al. SnoRNA U50 is a candidate tumor-suppressor gene at 6q14.3 with a mutation associated with clinically significant prostate cancer. Hum. Mol. Genet. 2008, 17, 1031-1042.

45. Grimson, A.; Srivastava, M.; Fahey, B.; Woodcroft, B.J.; Chiang, H.R.; King, N.; Degnan, B.M.; Rokhsar, D.S.; Bartel, D.P. Early origins and evolution of microRNAs and Piwi-interacting RNAs in animals. Nature 2008, 455, 1193-1197.

46. Thompson, J.D.; Gibson, T.J.; Plewniak, F.; Jeanmougin, F.; Higgins, D.G. The CLUSTAL_X windows interface: Flexible strategies for multiple sequence alignment aided by quality analysis tools. Nucleic Acids Res. 1997, 25, 4876-4882.

47. Bailey, T.L.; Elkan, C. Fitting a mixture model by expectation maximization to discover motifs in biopolymers. Proc. Int. Conf. Intell. Syst. Mol. Biol. 1994, 2, 28-36.

48. Gupta, S.; Stamatoyannopoulos, J.A.; Bailey, T.L.; Noble, W.S. Quantifying similarity between motifs. Genome Biol. 2007, 8, R24.

49. Matys, V.; Fricke, E.; Geffers, R.; Gossling, E.; Haubrock, M.; Hehl, R.; Hornischer, K.; Karas, D.; Kel, A.E.; Kel-Margoulis, O.V.; et al. TRANSFAC: Transcriptional regulation, from patterns to profiles. Nucleic Acids Res. 2003, 31, 374-378.

50. Yang, J.H.; Zhang, X.C.; Huang, Z.P.; Zhou, H.; Huang, M.B.; Zhang, S.; Chen, Y.Q.; Qu, L.H. snoSeeker: An advanced computational package for screening of guide and orphan snoRNA genes in the human genome. Nucleic Acids Res. 2006, 34, 5112-5123.

51. Gardner, P.P.; Daub, J.; Tate, J.G.; Nawrocki, E.P.; Kolbe, D.L.; Lindgreen, S.; Wilkinson, A.C.; Finn, R.D.; Griffiths-Jones, S.; Eddy, S.R.; et al. Rfam: Updates to the RNA families database. Nucleic Acids Res. 2009, 37, D136-D140.

52. Lestrade, L.; Weber, M.J. snoRNA-LBME-db, a comprehensive database of human H/ACA and C/D box snoRNAs. Nucleic Acids Res. 2006, 34, D158-D162.

53. Hofacker, I.L.; Fontana, W.; Stadler, P.F.; Bonhoeffer, L.S.; Tacker, M.; Schuster, P. Fast folding and comparison of RNA secondary structures. Monatsh. Chem. 1994, 125, 167-188.

54. Thompson, J.D.; Higgins, D.G.; Gibson, T.J. CLUSTAL W: Improving the sensitivity of progressive multiple sequence alignment through sequence weighting, position-specific gap penalties and weight matrix choice. Nucleic Acids Res. 1994, 22, 4673-4680.

55. Tamura, K.; Stecher, G.; Peterson, D.; Filipski, A.; Kumar, S. MEGA6: Molecular evolutionary genetics analysis version 6.0. Mol. Biol. Evol. 2013, 30, 2725-2729.

56. Ronquist, F.; Huelsenbeck, J.P. MrBayes 3: Bayesian phylogenetic inference under mixed models. Bioinformatics 2003, 19, 1572-1574. 
57. Posada, D.; Crandall, K.A. MODELTEST: Testing the model of DNA substitution. Bioinformatics 1998, 14, 817-818.

58. Hasegawa, M.; Kishino, H.; Yano, T. Dating of the human-Ape splitting by a molecular clock of mitochondrial DNA. J. Mol. Evol. 1985, 22, 160-174.

59. Graham, F.L.; van der Eb, A.J. A new technique for the assay of infectivity of human adenovirus 5 DNA. Virology 1973, 52, 456-467.

(C) 2015 by the authors; licensee MDPI, Basel, Switzerland. This article is an open access article distributed under the terms and conditions of the Creative Commons Attribution license (http://creativecommons.org/licenses/by/4.0/). 